\title{
La pobreza persistente como un fenómeno situado. Notas para su abordaje
}

Persistent Poverty as a located phenomenon. An approach to its reversal

Adriana Clemente

Docente e investigadora de la Facultad de Ciencias Sociales, UBA

aclemente@arnet.com.ar

Fecha de recepción:

24.10.15

Fecha de aceptación:

13.2.16

\section{Resumen}

Este artículo aborda el tema de la pobreza urbana en su expresión territorial. Su objetivo es brindar elementos conceptuales para la revisión de enfoques y estrategias de abordaje de la pobreza persistente y de su reversión por medio de políticas sociales de carácter universal. Para esto el trabajo toma aspectos de orden conceptual que revisan el abordaje y convenciones sobre la pobreza como categoría de análisis e intervención. En este sentido se aporta una suerte de caracterización de los enfoques dominantes y principales debates sobre la orientación de los programas sociales en las dos últimas décadas. En un segundo momento el texto desarrolla orientaciones sobre el enfoque situacional del abordaje de la pobreza urbana, identificando atributos de las políticas para actuar en los territorios complejos como son las villas y asentamientos más pobres. Finalmente se hace un avance sobre la problemática de accesibilidad, como un eslabón clave en el proceso de implementación de las políticas sociales que se proponen llegar a los hogares que padecen pobreza persistente y que tienen como particularidad una alta dependencia del Estado para atender sus necesidades más básicas.

Palabras clave: pobreza persistente - territorios urbanos - política social - integralidad - accesibilidad. 


\begin{abstract}
This article approaches urban poverty in its territorial expression, aiming at the provision of conceptual elements for acritical revision of current conceptual perspectives and strategies in order to reverse persistent poverty through universal social policies. In the first section we review the main conceptual categorizations which characterized the policy debates during last two decades. In the second one the central features of the situational approach to urban poverty are presented, focusing on the main attributes of policies implemented in complex territories such as "villas" and poor settlements. Finally, we explore questions of accessibility as a key issue to the implementation of social policies, including access to households in persistent poverty and a strong dependency from state agencies in order to fulfill their basic needs.
\end{abstract}

Key-words: persistent poverty - urban territories - social policy - integrality - accessibility

\title{
Introducción ${ }^{1}$
}

Este artículo aborda el tema de la pobreza urbana en su expresión territorial. De manera específica nos interesa profundizar sobre un tipo particular de pobreza que experimentan algunos hogares y definimos como persistente por tratarse de una condición de privación generalizada y extendida en el tiempo, donde - a partir de la privación económica- se combinan críticamente indicadores deficitarios tanto del hogar como de su entorno. Su particularidad es que las privaciones más urgentes tienden a mantenerse en el tiempo y comprometen a más de una generación de un mismo grupo familiar, aun a pesar de cambios favorables en su contexto económico, lo que significa un desafío para las políticas sociales (Clemente 2014).

Desde el punto de vista estadístico la pobreza persistente queda comprendida dentro del universo de la indigencia, donde el principal indicador es el bajo ingreso y no las múltiples derivaciones asociadas al mismo. La salida de la pobreza masiva que vino experimentando la sociedad argentina en la última década impone la evaluación de cómo ha impactado la reducción de la pobreza en los enclaves territoriales urbanos (villas y asentamientos) donde las instituciones tradicionales de la política social no logran el impacto esperado con las familias más pobres. Estas políticas vienen operando en es- 
cala y para un sujeto genérico que demanda alguna solución de modo sectorial (salud, educación, cuidado infantil, etc.). La universalidad requiere de acciones que complementen su poderosa acción, especialmente cuando se trata de soluciones que requieren intervenciones altamente profesionalizadas y con ingenierías específicas. Es el caso de los programas de terminalidad educativa, rehabilitación de adicciones, empleabilidad de personas con baja nivel educativo, programas que actúan con algún grado de especialización en torno a uno o más problemas específicos de la pobreza cuya solución puede marcar el cambio de esa condición. ${ }^{2}$

Al respecto postulamos que a pesar de la drástica reducción de la demanda masiva y urgente (alimentos y abrigo) las instituciones de la política social, particularmente las asistenciales continuaron actuando bajo la lógica de la prestación por demanda espontánea, propia del peor momento de la crisis de fin de siglo y no avanzaron suficientemente sobre los daños derivados de esa crisis. De ahí la importancia de problematizar sobre el modo de actuar con la población afectada por las crisis de inicio de siglo. De acuerdo a las estadísticas la población más afectada es la de jóvenes adultos varones y mujeres con bajo nivel educativo y calificación laboral, jefas y jefes de hogar atravesados por problemas de salud y déficit habitacional, familias numerosas cuyas redes de solidaridad también están dañadas.

Este trabajo se estructura en tres partes principales, una de orden conceptual que revisa el abordaje y convenciones sobre la pobreza como categoría de análisis e intervención, una segunda parte propone una suerte de clasificación sobre las improntas que toman las políticas en su enfoque y finalmente, se hace un avance sobre la perspectiva situacional de las políticas de asistencia directa y de su accesibilidad para los sectores más pobres.

\section{La pobreza como objeto de análisis e intervención}

En este artículo proponemos hablar de la pobreza como un fenómeno situado, esto es que lo que se defina como una condición de pobreza, al momento de pensar su abordaje supone, además de un marco conceptual y de la identificación de las causas de orden estructural que la explican, reconocer causas y atributos de orden micro social que se materializan en territorios concretos y terminan de amalgamar el modo en que se expresa el problema de la pobreza y su reproducción.

En tal sentido, el carácter situacional que adquiere la pobreza urbana en territorios concretos y aparentemente homogéneos (villas, asentamientos, complejos habitacionales, etc.) explica por qué una misma estrategia de la política social puede obtener resultados muy diferentes según su contexto final de aplicación.

Una perspectiva situacional del abordaje de la pobreza debe considerar los aspectos históricos y culturales como parte de la definición del problema y de su potencial abordaje; 
de ese modo evitamos la tentación de hacer comparaciones reduccionistas entre sociedades con tradiciones muy diferentes, especialmente en lo que refiere a la historia de las conquistas sociales y el rol del Estado en la cuestión social.

La pobreza como categoría de análisis, tiene las debilidades propias de ser un concepto que remite a un fenómeno dinámico, sujeto a permanentes cambios por efecto de otros factores asociados (cambios políticos, económicos, catástrofes, etc.).Los diferentes puntos de partida y el modo particular en que cada sociedad define "qué es ser pobre" es motivo de permanente revisión en un juego de expectativas donde la historia de reivindicaciones de una sociedad y los acuerdos universalizantes se ponen en tensión. ${ }^{3}$

Es en torno al carácter cambiante y polisémico del concepto que se hacen observaciones críticas sobre el sustento teórico que tiene la pobreza como categoría analítica. Gonzalez (1999) se refiere al concepto de pobreza como un concepto "altamente proliferante" en alusión a que no pertenece a ningún cuerpo teórico en particular y tiene a su alcance la capacidad de ingresar problemáticamente a los más diversos ordenamientos conceptuales.

Por su parte Scribano (2000) habla del mundo de la pobreza y de la representación que ese mundo genera, a nivel de su percepción, y que lo vincula con otros fenómenos como violencia y conflicto social. En consecuencia la intervención sobre la pobreza no escapa a lo que Scribano llama "proceso de eufemización etiquetante" en alusión al proceso institucional que va desde la simple identificación del actor o la familia pobre y termina con su estigmatización.

Scribano cita las metáforas a las que se recurre en los estudios sobre el tema y establece lo que llama tres grandes grupos de "mediaciones metafóricas". En primer lugar las que recurren a la imagen bélica (combatir, luchar, eliminar). En segundo lugar, las analogías médicas, tales como extirpar o mitigar y en tercer lugar, las geológicas /naturales que utilizan nociones como zonas, bolsones, estratos, desplazamientos. El autor concluye en que el conjunto de metáforas (en cualquiera de sus tres variantes) remite a conflicto, enfermedad y fenómeno natural. En esta línea de interpretación, podemos agregar que hoy se impone la noción de "desventaja/oportunidad", para referir la posición del sujeto pobre en la sociedad y se le atribuye la posibilidad de desenvolverse (en mayor o medida) en torno a las denominadas "estructuras de oportunidades". ${ }^{4}$

En cuanto al conocimiento científico que se produce en torno al fenómeno de la pobreza es posible afirmar que la investigación social participa activamente de la construcción de la pobreza como un campo polisémico, donde aún se libran debates sobre la virtud de un método u otro de medición, observándose como una falencia la falta de mediaciones entre el abordaje estadístico y el micro social. 
Como antecedente de estas mediaciones, en la Argentina contemporánea se pueden señalar algunos estudios emblemáticos como El País de los Excluidos (CIPPA 1991); financiado por la Interamerican Foundation (IAF), tomó testimonios (por la vía de grupos focales) en barrios pobres de diferentes zonas del conurbano bonaerense, en torno a cuatro áreas temáticas: hábitat y participación, salud, empleo y educación. El informe fue confeccionado por un grupo de especialistas en temas económicos y sociales, algunos de los cuales ya habían participado en el emblemático estudio La pobreza en Argentina. ${ }^{5}$ Se trató de trabajos que en plena recuperación democrática también apelaban a la necesidad de que el Estado recuperara su iniciativa en materia social.

El aporte de Minujin (1992) en Cuesta Abajo ocupa un lugar central en la bibliografia sobre el tema. Se trata del primer texto que abordó el empobrecimiento de la clase media y acuñó la noción de "nuevos pobres". Este texto, compuesto por un conjunto de capítulos a cargo de diferentes especialistas, puso en crisis la conceptualización clásica del pobre y la pobreza como un fenómeno inherente a la marginalidad.

En lo referido al abordaje conceptual de la pobreza en la región, Leguizamón (2008) indaga sobre los antecedentes empíricos y conceptuales de los procesos socio-históricos y estructurales de más larga data que llevan a la producción de la pobreza masiva. ${ }^{6}$ La autora diferencia producción y reproducción de la pobreza, atribuyendo a la producción el estudio de las causas y a la reproducción el de los factores contingentes o coyunturales que la mantienen y/o incrementan. Para Leguizamón la reproducción tiene que ver más con los aspectos coyunturales vinculados a la dinámica económica de la acumulación de la riqueza (precarización del mercado de trabajo, devaluaciones, etc.). Esta caracterización deja intencionalmente fuera la perspectiva subjetivista que explica la pobreza más por efecto de las trayectorias particulares que por problemas estructurales.

De la revisión de los trabajos académicos también se puede estimar que los más significativos que tienen a la pobreza como objeto de estudio, son mayoritariamente descriptivos, tanto cuantitativos como principalmente cualitativos, que abordan el fenómeno para explicar "otro" problema asociado en materia de vivienda, salud y/o trabajo, donde la pobreza es tomada como el contexto del problema y no como un fenómeno producido que lo constituye en un objeto de estudio en sí mismo.

\section{Sobre las justificaciones y la intervención en torno a la pobreza}

La debilidad conceptual que subyace a la definición de pobreza, se traslada al campo de la intervención social y explica cuestiones como las "recetas" que buscan respuestas universales a los problemas sociales la mayoría de las veces, sin considerar las determinantes sociales, políticos e históricos de esos problemas. ${ }^{7}$ Postulamos que la problematización de la pobreza como cuestión de Estado depende de la intencionalidad de producir al- 
guna transformación real del problema que explica la persistencia de la pobreza. En tal sentido, las instituciones de la política expresan a través de su especificidad (funciones, competencias, presupuesto) la particular atención que se le otorga a la pobreza como un problema de la sociedad o como un problema de los "pobres". ${ }^{8}$

A modo de ensayo y tomando en cuenta la evidencia que arroja el análisis de los programas sociales y su justificación en la historia reciente, es posible ordenar el universo en torno a tres concepciones dominantes y en muchos casos con límites difusos entre sí: a. pobreza como externalidad del modelo de mercado, b. pobreza como amenaza de la convivencia social, c. pobreza como vulneración de derechos. Esta suerte de clasificación de cómo las instituciones de la política social interpretan y en consecuencia organizan sus prestaciones, propone dar cuenta de tendencias ya que la convivencia de paradigmas es común y se explica por la naturaleza pragmática de las políticas sociales. Es así que en el esquema también aparece contextuada la gerencia social, tema que nos ocupa en este trabajo. Los ejes identificados explican la pobreza como: externalidad del modelo, como amenaza y pobreza como vulneración de derechos (Clemente 2014):

a. La pobreza como externalidad del modelo ha sido el enfoque dominante durante las reformas neoliberales y se sustenta en una lógica conservadora, donde la pobreza es una externalidad del modelo de mercado y las correcciones apuntan a regular los desfases. Desde el punto de vista institucional este planteo también alimenta la hipótesis de que la acción del Estado es ineficiente y que la pobreza se explica, más que por cuestiones económicas, por una "mala coordinación” y aplicación del gasto por parte del Estado. La perspectiva de la pobreza como una externalidad de la economía sostiene a la gerencia social como modelo de intervención correctivo y eficiente. Se trasladan al Estado los mismos parámetros de eficiencia que se le pide a la empresa.

Desde el punto de vista práctico el modelo se apoya en un planteo de co- responsabilidad entre el que provee el recurso (estado, ONG, iglesia, etc.) y el beneficiario. Desde esta perspectiva se otorga cierta condición de inevitabilidad de la pobreza que se presenta como un "ciclo fatídico de auto-reproducción". Así la salida por efecto de alguna oportunidad de uno o más miembros es la clave para la superación del problema, negando así el carácter colectivo y redistributivo que tienen los modelos que en la historia, han demostrado resultados en la reducción de las desigualdades sociales. El abordaje territorial ha sido un modus operandi de este enfoque, no tanto para actuar en las causas sino en los efectos del problema y su contención.

b. La pobreza como amenaza es el enfoque clásico que inspiró la Ley de Pobres (1887) en plena revolución industrial y que, aunque actualizado, mantiene vigencia. Desde esta perspectiva la pobreza es una "posición” que se ocupa por que otorga algún beneficio. En este caso domina la idea de "auto reproducción" por responsabilidad de los indivi- 
duos que la padecen. De este modo se justifican acciones del Estado de aislamiento y penalización (encierro, institucionalización, judicialización) y segmentación de la oferta (calidad y cobertura) de servicios sociales (educación, salud, cuidados infantiles, etc.). Esta perspectiva se traduce en programas donde el componente normativo, bajo diferentes formatos, es el que tiene mayor peso en el diseño. En varios aspectos esta orientación se articula, ideológicamente, con el enfoque gerencial, solo que opera más fuertemente sobre los aspectos de control $y$ disciplinamiento que se pueden ejercer desde el sistema institucional de aplicación de la política social. El modelo "tutelar" es el que mejor representa esta lógica que asocia la pobreza a la violencia, la enfermedad y el delito.

c. La pobreza como vulneración de derechos, supone ubicar al Estado como garante del bienestar colectivo. Es así que se involucra a la política y a la sociedad en la construcción de la respuesta que por concepto va a representar alguna disputa de poder por la distribución secundaria de la riqueza. ${ }^{9}$

Como es de suponer, estas tres lógicas no se presentan de modo puro y pueden convivir dentro de un mismo sistema y sin resolver sus tensiones. Sin embargo, el enfoque de los derechos sociales, por su naturaleza incremental es el que presenta mayores resistencias en muchos sectores de la sociedad, aún en sectores medios que adoptan posiciones conservadoras frente a los procesos de ampliación de derechos de los que ellos mismos resultan beneficiados. De cualquier modo es posible acordar que la perspectiva gerencial resulta dominante por sobre la de derechos sociales y que incluso se presenta correctiva para asegurar el enfoque de derechos en tanto búsqueda de equidad y eficiencia como garantía del cumplimiento de esos derechos.

\section{Sobre la construcción de lo situacional en el abordaje de la pobreza}

Como ocurre en otros órdenes de la sociedad, los discursos dominantes tienen consecuencias prácticas respecto del fenómeno sobre el que predican. De ahí la importancia de identificar a los actores que participan de la construcción del discurso público sobre la pobreza (causas y derivaciones), especialmente si sus especificaciones habilitan y/o impulsan nuevas intervenciones, tales como: subsidios, cambios de legislación, reducción o aumento del gasto social, etc.

Según lo que se ha podido avanzar en el conocimiento y conceptualización de la pobreza y su persistencia en el contexto urbano, el factor territorial es una parte determinante del problema y su expresión. Aludimos por factor territorial a la configuración que adquiere "lo comunitario" ya no sólo como lugar de encuentro identitario y fuente del lazo social, sino también como ámbito de disputa y confrontación de intereses y reproducción de nuevas desigualdades. Es en este sentido que la perspectiva redistributiva de la política social, en el momento de intervenir, debe ser situacional, 
atendiendo al carácter político y los elementos de reproducción que se presentan en los territorios, especialmente donde hay enclaves de pobreza y marginación social, ya que es ahí donde la falta del poder regulatorio que puede ejercer el Estado deja a la población más vulnerable aún más expuesta a las fuerzas especulativas que pueden estar interviniendo.

La importancia de "situar" problemáticas refiere a la necesidad de encontrar una interacción entre las políticas de tipo universal, los subsidios monetarios y otras estrategias de la política social que se instrumentan con un enfoque de derechos sociales. Se trata de recursos que buscan impactar y producir transformaciones en las condiciones de vida de un conjunto de familias, cuya situación de pobreza extrema las pone en una situación de mayor vulnerabilidad que otros habitantes del mismo barrio. De ahí que hablamos de intervenciones situadas. Se busca superar discusiones estériles que ponen en oposición el enfoque universal con el focalizado, como si fueran antagónicos, cuando en la práctica y según el problema que se aborda, pueden actuar de modo complementario.

El conjunto de dimensiones a considerar para abordar la pobreza como una problemática situada supone la combinación de diferentes aspectos de la realidad que se espera transformar, donde la dimensión individual se expresa en la situación de los miembros del hogar (según parámetros estadísticos), mientras que la dimensión colectiva se materializa en el entorno de estos hogares y su acceso a bienes, servicios, fuentes de trabajo, etc. Es la dimensión colectiva y territorial la que a nuestro criterio termina de "situar" el tratamiento del problema de las familias más pobres, pues en ese entorno hay claves que operan en la persistencia o no de esa condición. ${ }^{10}$

Por el carácter multidimensional del problema y según las conclusiones del estudio sobre pobreza persistente que da fundamento a este artículo, en el plano de la intervención es posible establecer que las líneas programáticas que tengan por objeto los hogares cuyos miembros sufren condiciones de pobreza y/o indigencia deberían prever insumos en torno a por lo menos tres dimensiones implicadas en el desenvolvimiento diario de los miembros del hogar y su reproducción: las condiciones ambientales del hogar y su entorno (hábitat familiar y comunitario), la dinámica de los vínculos intrafamiliares y extra muros y los resultados de las prácticas de aprovisionamiento, que comprenden a un conjunto de desempeños que realizan los grupos convivientes para sustituir los ingresos que no posibilita el mercado de trabajo (formal e informal) dejando a la economía familiar sujeta a un proceso de creciente des-mercantilización y en franca dependencia de la política de subsidios y de ayuda social que instrumentan las redes territoriales de asistencia. Estas dimensiones cobran diferente peso y significado según la situación de la familia, la ecuación es que el peso del entorno crece en la medida que disminuyen los ingresos provenientes del mercado. ${ }^{11}$ 
La perspectiva situacional de la planificación (Matus, 1975) llevada al campo de la intervención social, pondera el carácter político que adquiere la planificación del desarrollo a partir de cómo interactúan los actores implicados en esos procesos que son: los técnicos, los políticos, la burocracia y los grupos sociales organizados. Sus interacciones no pueden analizarse por fuera del contexto en el que se producen la relación de cooperación y oposición según corresponda a sus intereses. ${ }^{12}$

A los fines de avanzar en una perspectiva situacional del abordaje de la pobreza, postulamos que la pobreza como objeto de intervención es una condición donde los aspectos mensurables (estructura del grupo, situación de la vivienda, ingresos, nivel educativo, etc.) deben ampliarse con los aspectos vinculares de mayor intangibilidad (lazos afectivos, prácticas de cuidado, participación social, etc.). Resultando el territorio el ámbito donde se desarrolla el ciclo de vida de la familia y en consecuencia, el lugar de posibilidad para que las dimensiones materiales y vinculares, se combinen y potencien los resultados de la batería de políticas que tienen a las familias como foco de su acción.

De ahí la importancia de que las intervenciones destinadas a las familias en situación de pobreza estructural e indigencia comprendan que sus resultados están sujetos a otros factores de contexto y en la medida que no se actúe sobre esos otros factores, ellos adquieren rango de factores determinantes. Al respecto podemos señalar la tugurización, el hacinamiento en el hogar, la ruptura de vínculos de confianza parentales y de vecindad; la baja calidad de las prestaciones sociales que llegan a estas familias actúan como factores asociados para reducir el impacto de las políticas (particularmente las de transferencia monetaria) que tienen como meta reducir la pobreza y las desigualdades.

Se trata de familias con alta dependencia de su entorno y de las políticas de ayuda social. La existencia o no de redes de contención en su entorno también definen la situación de estas familias. Las redes pueden actuar en clave de cooperación y ayuda mutua, como así también en clave de abuso o nuevos daños. Las redes de cooperación son las que favorecen que la familia en situación de extrema pobreza se pueda beneficiar con el apoyo y la mediación de redes de ayuda y cuidado. De manera contraria las relaciones de abuso refieren a redes que se organizan en torno a prácticas de riesgo, particularmente delictivas y/o abusivas y violentas tanto de pares como de las fuerzas de seguridad (maltrato, desalojos compulsivos, extorsiones, etc.). En definitiva la evidencia demuestra que a mayor pobreza, mayor es la posibilidad de sufrir diferentes tipos de violencias -domésticas, comunitarias y represivas- (Elizalde y Miglioni 2013).De ahí la necesaria comprensión diagnóstica del hábitat (características físicas, conflictividad social, etc.) en la composición situacional de los enclaves de pobreza persistente.

Se trata de reconocer las situaciones en su contexto y así evitar generalizaciones bajo la suposición de los recursos de la política social, cuando están concebidos bajo el pa- 
radigma universal, llegan a todos los hogares del mismo modo. La noción de abordaje situacional es un enfoque orientado a consignar que el diseño de las políticas que esperan transformar situaciones de pobreza persistente y/o prevenirla deberá atender las particularidades del contexto. Según la experiencia acumulada con los programas de transferencia de ingresos, hay que relativizar el impacto de las intervenciones que pretenden tener resultados totalizadores a partir de una única estrategia. Es justamente en la heterogeneidad que se sustenta la necesidad de enfoques que actúen en las desigualdades y brechas que también se reproducen muy fuertemente a nivel micro social. ${ }^{13}$

Las instituciones de la política social se expresan a través de su especificidad (funciones, competencias, presupuesto, etc.). Así las formas para acceder a las prestaciones sociales y la calidad de las soluciones son, entre otros aspectos, indicios de cómo se define el problema. De ahí la importancia de los atributos que se prevén en los programas destinados a llegar a los enclaves territoriales (villas, asentamientos, complejos habitacionales tugurizados, etc.) sin reproducir las desigualdades; también se expresan en los asentamientos cuya población comparte en apariencia las mismas condiciones de vulnerabilidad. Así aspectos diferenciados de accesibilidad a los bienes y servicios públicos, la calidad de la oferta (segmentada o no) que llega desde el Estado a esta población, y la presencia o no de redes que vinculen a las comunidades y las familias con el Estado son algunas de las claves que hacen a la composición final de las estrategias que deben considerar el peso del territorio y las disputas por su control, que actúan de modo negativo en la revinculación de las familias más pobres con el Estado. ${ }^{14}$

La accesibilidad es un capítulo destacado tanto para el diseño de las soluciones, como principalmente para el proceso de implementación de las políticas destinadas a los sectores más pobres, que también son los que tienen más dependencia de los servicios y recursos que brinda el estado. Por tal motivo la accesibilidad se convierte en un tema central para estudiar y evaluar los resultados de las políticas sociales.

La noción de accesibilidad como vínculo que se construye entre los sujetos y los servicios responsables de materializar las políticas sociales (Stolkiner 2000, Carballeda 2012, Elizalde y Maglioni 2013) supone por concepto encuentros y desencuentros entre las prestaciones y sus ingenierías con relación a los destinatarios y sus expectativas. El análisis que se viene haciendo de la problemática supone la necesidad de captar tanto los procesos como los escenarios donde se dan esos procesos. El proceso indica algún tipo de secuencia, un trayecto que se produce en la interacción entre los sujetos que experimentan las necesidades y las instituciones que prestan servicios en consonancia con las mismas. El escenario refiere a las condiciones (físicas, económicas, normativas) en las que se produce la interacción y se determinan las condiciones en que se dan la relación entre sujeto, la institución y sus servicios. Entonces, el análisis de la accesibilidad de las políticas, particularmente de los programas de asistencia directa, requiere tanto un 
enfoque diacrónico referido al proceso y su evolución temporal, como sincrónico para captar el modo en que el escenario condiciona ese proceso.

El estudio sincrónico de la accesibilidad es el más complejo, pues comprende el conocimiento de la convergencia de prácticas que desarrollan los diferentes actores que forman parte del proceso que va desde el diseño de la política a su concreción, cuando las prestaciones (directas e indirectas) se materializan (Clemente 2014). Los responsables del diseño anticipan una solución a necesidades identificadas; a su vez las personas portan expectativas que no siempre se corresponden con esa solución. Al mismo tiempo están los mediadores, cuya acción puede ampliar o estrechar la brecha (potencial) entre solución y expectativa. El mediador actúa desde dos marcos institucionales de complementación posible. Por un lado están los actores sociales (organizaciones, movimientos sociales, ONGs, partidos políticos, etc.) y los efectores institucionales. Estos últimos comprenden un amplio espectro de perfiles que van desde el portero de un colegio, hasta un médico o un trabajador social. Son efectores de la política social que en el ejercicio de su función institucional terminan de direccionar el resultado final de una política social -de ahí su importancia-.

En tal sentido, según lo expresado desde lo que llamamos enfoque de intervención situacional sostenemos que el contexto social y económico de los hogares más pobres y el desempeño de las instituciones que los asisten se combinan con resultados no siempre comparables. Esta dificultad para llegar con los mismos estándares a los grupos más pobres, explicaría por qué la universalidad como enfoque de la política social, especialmente la de asistencia directa, alcanza resultados diferentes, aun bajo condiciones (sociales, económicas e institucionales) en apariencia equivalentes.

\section{Nota final}

Además de revisar los vaivenes que ha tenido el tratamiento contemporáneo de la pobreza como categoría de análisis, este artículo propone repensar las implicancias metodológicas que tienen sus definiciones a la hora de intervenir, especialmente en medios urbanos tugurizados y con la población más pobre. En general, se espera que las transferencias monetarias tengan un impacto significativo y rápido a la hora de revertir las desigualdades, pero esta expectativa no se condice con la lenta y heterogénea reacción que pueden desplegar las familias pobres, especialmente las afectadas por condiciones de pobreza persistente. Este trabajo es un aporte preliminar para pensar el modo en que los enfoques dominantes respecto de la pobreza (generalmente monetaristas) que tienden a homogenizar el fenómeno y su tratamiento. La propuesta de abordaje situado que esbozamos en este artículo recupera lo mejor de la tradición de trabajo social comunitario antes de que las reformas neoliberales capitalizaran las estrategias de "proximidad" y se ponderara (de modo encubierto) a la focalización como método de contención en 
tanto se producían las reformas y el vaciamiento del Estado. Sin embargo reconocemos que superada la pobreza masiva, se presentan situaciones donde algunos hogares quedan atrapados y con el único auxilio del Estado como alternativa para revertir su situación. De ahí la importancia de evaluar cómo llega el Estado y sus recursos a estas familias y los enclaves donde ellas habitan. 
${ }^{1}$ Este artículo reproduce algunos de los principales resultados de la investigación "Familiarización del enfoque de bienestar, últimas redes y reconfiguración de la pobreza y la indigencia en medios urbanos. UBACyT/ PIUBAMAS 04 (2010-2013), así como avances del Proyecto Reconfiguración de los regímenes de bienestar pos-neoliberal y de la pobreza persistente en América Latina (UBACyT/ PIUBAMAS 04 (2013-2016). Ambos proyectos co dirigidos por la autora de este artículo.

${ }^{2}$ En la segunda presidencia de Cristina Fernández de Kirchner (2011-2015) se debe destacar el caso del Programa de terminalidad educativa FINES ejecutado por el Ministerio de Educación de la Nación y el Programa Ellas Hacen destinado a mejorar la empleabilidad de las mujeres que ejecuta el Ministerio de Desarrollo de la Nación, así como las primeras experiencias de abordaje interministerial que impulsó el Consejo Nacional de Políticas Sociales (Programa Ahí). Al momento de elaborarse este artículo se está produciendo el cambio de gobierno y se desconoce la continuidad o no de estas políticas.

${ }^{3}$ Es el caso de las Metas del Milenio. Metas que para Argentina y Uruguay, por ejemplo, no agregan demasiado desafio por su nivel de desarrollo, mientras que para otros países de América Latina, con menor desarrollo, sí lo representan.

${ }^{4}$ Las estructuras de oportunidades se definen como probabilidades de acceso a bienes, a servicios o al desempeño de actividades. Este enfoque afirma que los recursos que controlan los hogares no se pueden valorar con independencia de las oportunidades a las que tienen acceso. Esas oportunidades son el resultado de un entramado entre la acción del mercado y la del Estado.

${ }^{5}$ En 1984, la nueva dirección del INDEC asumió como compromiso la tarea de dar cuenta de manera estadística de la situación social que se "heredaba" de la dictadura. Con el apoyo técnico de la CEPAL, Oscar Altimir dirigió junto a A. Minujin y Horacio Somigliana el trabajo de procesamiento y análisis de los datos del Censo de 1980 de acuerdo a la metodología utilizada en Altimir (1979).

${ }^{6} \mathrm{La}$ autora refiere a que el concepto de pobreza masiva reemplaza al de pauperismo que cayó en desuso.

${ }^{7}$ Por ejemplo es el caso de comparar el impacto de los programas de transferencia de ingresos por la vía de sistemas no contributivos en países como Brasil, Argentina y Bolivia sin considerar el punto de partida de cada una de estas sociedades en cuanto a su grado de sindicalización, cobertura del sistema educativo, derechos sociales institucionalizados y estructura del mercado de trabajo entre otros.

${ }^{8}$ Siguiendo a Simmel (1908) y de modo contemporáneo a Paugam (2007) los pobres son una categoría social y no son quienes sufren las carencias y privaciones específicas, sino los que reciben auxilio o deberían recibirlo según las normas sociales. En este sentido es la ingeniería institucional lo que expresa esta concepción.

${ }^{9}$ En el caso argentino el ejemplo emblemático del enfoque de derechos lo inauguró la Fundación Evita (19481955). Bajo el lema propuesto por su fundadora: “Donde hay una necesidad hay un derecho", habilitó el tratamiento institucionalizado de las necesidades sociales como derechos. En clave contemporánea, subsidios de carácter universal como la Asignación Universal por Hijo (2009) implementado en el primer gobierno de Cristina Fernández de Kirchner (2007/2011) es un hito de este enfoque, por considerar la informalidad laboral de los padres como una condición de vulnerabilidad social de los niños y así equiparar sus derechos al de los niños cuyos padres están insertos en el mercado formal de trabajo.

${ }^{10}$ Para los propósitos de este trabajo, interesa destacar la noción de territorio, o mejor dicho territorialidad -que se refiere a la percepción que los sujetos tienen de su entorno con relación a diversos aspectos de su vida (hábitat, medio ambiente, acción social y procesos comunicativos). Las acepciones de dominio y espacio son inherentes a la noción de territorialidad (Montañés Gómez 1997).

${ }^{11}$ Llamamos redes territoriales de asistencia a la trama de organizaciones sociales, que aunque no estén vinculadas entre sí, constituyen un sistema que se articulan en torno a una o más políticas públicas actuando como mediadores entre los sujetos que padecen necesidades y los recursos de carácter gubernamental signados para ese fin (alimentación, abrigo, salud, etc.).

${ }^{12}$ La noción de situación desde la perspectiva que propone Carlos Matus (1978) remite a la relación que se establece entre el actor y la acción. Situación es todo aquello que es relevante para la acción; a su vez la situación es condicionante de la acción y la acción es eficaz en la construcción de la situación.

${ }^{13}$ El enfoque de abordaje integral como enfoque de la política social, fue propiciado por el Consejo de Políticas Sociales de la Nación a través del Ministerio de Desarrollo de la Nación entre 2012 y 2015. Se trata de un enfoque que actúa bajo la articulación de por lo menos tres tópicos: territorialidad, interdisciplinariedad e intersectorialidad entre ministerios.

${ }^{14}$ Hablamos de revinculación porque en este plano se produce un fenómeno donde, a pesar de alta dependencia de las ayudas sociales que provee el Estado, las personas que son objeto de los programas asistenciales suelen no otorgar una valoración positiva a dicho vinculo que según se ha podido observar se presenta como ocasional (aun cuando no lo es) y por momentos traumático y hostil a causa de los tiempos que insumen las gestiones, el carácter efimero de las respuestas o la calidad de los servicios que desde su percepción son insuficientes y/o inadecuados. 


\section{Referencias bibliográficas}

Altimir, Oscar (1979). La dimensión de la pobreza en América Latina. Santiago de Chile: CEPAL, serie Cuadernos http://bibliotecavirtual.clacso.org.ar/ar/libros/clacso/crop/ cattani/05legui.pd

Alvarez Leguizamón, Sonia (2009) "La producción de pobreza masiva y su persistencia en el Pensamiento social Latinoamericano". En Alberto D. Cimadamore y Antonio Cattani (coords.) La producción de pobreza en América Latina. Bogotá: Siglo del Hombre Editores/ CLACSO: 79-122.

Carballeda, Alfredo (2014) "La accesibilidad y las políticas de salud". Revista Sociedad. www.edumargen.org/docs/curso58-1/unid01/apunte02_01.pdf

CIPPA, Centro de Investigaciones sobre Pobreza y Políticas Sociales en la Argentina (1991) El país de los excluidos. Crecimiento y heterogeneidad de la pobreza en el conurbano bonaerense. Buenos Aires: CIPPA.

Clemente, Adriana (2014) "La pobreza como categoría de análisis e intervención". En Adriana Clemente (coord.), Territorios Urbanos y Pobreza Persistente. Buenos Aires, Espacio Editorial.

Elizalde, Carmen y Carolina Miglioni (2014). "Las redes como parte de la solución y como problema". En Adriana Clemente (coord.), Territorios Urbanos y Pobreza Persistente, cit.

Gonzalez, Horacio (1997) “El sujeto de la pobreza: un problema de teoría social”. En Alberto Minujin, Cuesta abajo. Los nuevos pobres: efectos de la crisis en la sociedad Argentina. Buenos Aires: UNICEF/Losada, págs.

Matus, Carlos (1975) Planificación de situaciones. México: Siglo XXI.

Minujin, Alberto, dir. (1992) Cuesta abajo. Los nuevos pobres: efectos de la crisis en la sociedad argentina. Buenos Aires: UNICEF/Losada.

Minujin, Alberto y Delamónica, Enrique(2007) “Infancia y Pobreza una discusión sobre conceptos y mediciones”. En Revista Medio Ambiente y Urbanización. Nro. 66.

Montañez, Gustavo (1997) Geografía y Ambiente: Enfoques y Perspectivas. Bogotá: Ediciones Universidad de la Sabana. 
Paugam, Serge (2007) Las formas elementales de la Pobreza. Madrid:Alianza Editorial.

Simmel, Georg (1908) "El pobre". En G. Simmel, Sobre la individualidad y las formas sociales. Introducción y edición por Donald N. Levine. Bernal, Pcia. de Buenos Aires: Universidad Nacional de Quilmes Ediciones 2002:118-146.

Stolkiner, Alejandra y Alicia Barcala (2000) "Reforma del sector salud y utilización de servicios de salud en familias con necesidades básicas insatisfechas". En: Jornadas Gino Germani. Buenos Aires: Instituto de Investigaciones Gino Germani, UBA, págs. 15-30 http://bibliotecavirtual.clacso.org.ar/ar/libros/argentina/germani/barcala.rtf

Scribano, Adrián (2002) “Pobreza, ciencias sociales y filosofia: hacia un análisis de los supuestos ontológicos de los estudios de pobreza”. Cuadernos No 15, Facultad de Historia y Ciencias Sociales, Universidad Nacional de Jujuy. http://www.scielo.org.ar/pdf/ cfhycs/n15/n15a09.pdf 